BMJ Paediatrics Open

\title{
Paediatric inflammatory multisystem syndrome temporally associated with COVID-19 (PIMS-TS): a narrative review and the viewpoint of the Latin American Society of Pediatric Intensive Care (SLACIP) Sepsis Committee
}

\author{
Jaime Fernández-Sarmiento (1D , ${ }^{1}$ Daniela De Souza, ${ }^{2}$ Roberto Jabornisky, ${ }^{3}$ \\ Gustavo Ariel Gonzalez, ${ }^{4}$ Maria del Pilar Arias López, ${ }^{5}$ Gladys Palacio ${ }^{6}$
}

To cite:

Fernández-Sarmiento $\mathrm{J}$ De Souza D, Jabornisky R, et al. Paediatric inflammatory multisystem syndrome temporally associated with COVID-19 (PIMS-TS): a narrative review and the viewpoint of the Latin American Society of Pediatric Intensive Care (SLACIP) Sepsis Committee. BMJ Paediatrics Open 2021;5:e000894. doi:10.1136/ bmjpo-2020-000894

- Additional material is published online only. To view, please visit the journal online (http://dx.doi.org/10.1136/ bmjpo-2020-000894).

Received 16 0ctober 2020 Revised 29 December 2020 Accepted 7 January 2021

Check for updates

(c) Author(s) (or their employer(s)) 2021. Re-use permitted under CC BY-NC. No commercial re-use. See rights and permissions. Published by BMJ.

For numbered affiliations see end of article.

Correspondence to Dr Jaime Fernández-Sarmiento; jaimefe@unisabana.edu.co

\section{ABSTRACT}

Background In this review, we discuss some important aspects of paediatric inflammatory multisystem syndrome temporally associated with COVID-19 (PIMS-TS), a new syndrome that is temporally related to previous exposure to SARS-CoV-2 infection. This virus has a broad spectrum of presentation that may overlap with Kawasaki disease in terms of presenting symptoms and laboratory and cardiac findings. Our objective was to review and summarise published evidence regarding the most important aspects of PIMS-TS, with special emphasis on the treatment strategies suggested for middle-income and low-income countries.

Methods A systematic review of the literature was performed in the principal medical databases including PubMed, Embase (OVID) and Google Scholar between December 2019 and August 2020.

Results A total of 69 articles were identified in the described databases. Altogether, 13 articles met the inclusion criteria and were eligible. The most frequently described symptoms of PIMS-TS include fever (82\%), shock (67\%) and gastrointestinal $(87 \%)$, skin $(71 \%)$ and cardiac disorders $(75 \%)$. In most series, it has been observed between 4 and 6 weeks after the pandemic appears in the general population. Multisystem inflammatory syndrome in children is presented as a great systemic inflammatory response syndrome, which sometimes presents as shock requiring fluid resuscitation and vasoactive drug support (26\%). Several treatment strategies have been used, including immunoglobulin, steroids, aspirin, anakinra and anticoagulation among others. These general and specific interventions should be guided by an interdisciplinary and multidisciplinary team, especially in settings with limited resources.

Conclusions PIMS-TS COVID-19 is a new type of presentation of SARS-CoV-2 infection, with an exaggerated inflammatory response and frequent-but not exclusivedigestive and myocardial involvement. It is important to describe the clinical course and outcomes in countries with limited resources as well as establish the role of biomarkers for early diagnosis, effective therapeutic strategies and outpatient follow-up schemes.

\section{What is known about the subject?}

Paediatric inflammatory multisystem syndrome temporally associated with COVID-19 (PIMS-TS) is a type of presentation of SARS-CoV-2 infection that produces a 10 times greater need for hospitalisation and mortality in children than other COVID-19 presentations.

- It is characterised by fever, shock and gastrointestinal, skin and cardiac involvement, with prior positive real-time PCR or antibody tests.

- The diagnostic and treatment approach should be aimed at initial stabilisation and shock management, especially in countries with limited resources. The specific treatment includes immunomodulators.

\section{What this study adds?}

For this SARS-CoV-2 disease, which mainly affects children, a comprehensive approach is suggested that may be applied under the various healthcare system access conditions, including strategies geared towards middle-income and low-income countries. This treatment includes general stabilisation and shock management measures as well as the specific immunomodulatory therapy currently recommended based on the available evidence.

\section{INTRODUCTION}

In December 2019, a new viral infection was reported, causing severe respiratory infection and very high mortality. According to its genetic sequencing, this virus belongs to the genus Beta coronavirus, closely related to the severe acute respiratory syndrome (SARS) virus. It was named SARS-CoV-2 and its disease COVID-19. ${ }^{12}$ 
Healthcare systems worldwide have been deeply concerned, given SARS-CoV-2's high transmissibility, severity and lethality, particularly in the population over the age of 60 years. ${ }^{1}$ Patients with major comorbidities such as heart disease, diabetes, hypertension or obesity have an increased risk of dying. ${ }^{12}$ Moreover, mortality has been associated with multiple organ failure as the common final pathway for pneumonia, sepsis and acute respiratory distress syndrome. COVID-19 is usually less severe in paediatric patients. In general, $80 \%-90 \%$ of children with SARS-CoV-2 infection are asymptomatic or have a mild infection. However, between $4 \%$ and $10 \%$ of hospitalised children may need to be transferred to a paediatric intensive care unit (PICU), and mortality ranges from $0.1 \%$ to $8 \% .{ }^{34}$ Recently, the Critical Coronavirus and Kids Epidemiology study reported a mortality rate of $5 \%$ in children hospitalised in critical care in five European and American countries (Chile, Colombia, Italy, Spain and USA), with $76 \%$ of cases having severe pneumonia as their main manifestation. ${ }^{4}$

Several pathophysiological factors may explain these features. COVID-19 non-survivors have higher serum ferritin, D-dimer and $\mathrm{C}$ reactive protein (CRP) than those who survive, indicating an intense inflammatory response. ${ }^{2}$ Recently, a new type of presentation of SARS-CoV-2 infection has been described in children, involving this significant inflammatory response. This new disease has been called paediatric inflammatory multisystem syndrome temporally associated with COVID-19 (PIMS-TS), a new syndrome that is temporally related to previous exposure to SARS-CoV-2 infection. This is a severe presentation of the virus in children and requires early detection to avoid its progression and potentially unsatisfactory outcomes. ${ }^{5}$ In this article, we discuss and review the most relevant aspects of PIMS-TS described to date.

\section{METHODS}

\section{Search strategy and article selection}

A systematic review of the literature was performed in the principal medical databases including PubMed, Embase (OVID) and Google Scholar, using the Medical Subject Headings (MeSH) terms ("SARS-CoV-2" OR "Covid-19" OR "coronavirus" OR "infection" OR "sepsis" OR "Covid-19" OR "critical care") AND "Multisystem Inflammatory Syndrome in Children" OR "MIS-C" OR "PIMS-TS" between December 2019 and August 2020. The descriptors were validated in descriptors in health science and $\mathrm{MeSH}$. Grey literature or as yet unpublished documents were not included.

\section{Eligibility criteria}

Articles that reported at least five cases of PIMS-TS, including case series, case reports, and cross-sectional, case-control, cohort (either prospective or retrospective) or clinical trial studies, were included. Studies of critically ill children with COVID-19 were also considered, and the cases of PIMS-TS reported in these studies were explored. Other inclusion criteria were articles that described important outcomes such as mortality, complications, laboratory findings and treatment received. Only articles in English, Spanish or Portuguese were considered. No reports of PIMS-TS in low-income and middleincome countries were found in indexed journals. The WHO, Centers for Disease Control and Prevention (CDC) and Royal College guidelines were consulted for the definitions. Articles that did not provide complete data when reporting general cases of critically ill children with COVID-19, or those for which the full text was not available, as well as narrative reviews, were excluded. Adult cases have already been described, but these were not included in this review.

\section{Study selection and data collection process}

First, the inclusion and exclusion criteria for this systematic review were defined, after which one of the researchers (JF-S) performed the systematic search of the literature and reviewed the most relevant articles. The established criteria were applied, and the articles were approved by all the Latin American Society of Pediatric Intensive Care (SLACIP) sepsis committee authors. In case of doubt, or a lack of consensus regarding the inclusion of an article, a second reviewer (RJ) was consulted to decide. Any discrepancies or missing data were resolved by consensus. Preferred Reporting Items for Systematic Reviews and Meta-Analyses guidelines were followed (figure 1).

\section{Patient and public involvement statement}

No patients participated actively in this review. The data were taken from the most important publications to date on PIMS-TS, including consensus recommendations for high-income countries. It is expected that this information will be disseminated through SLACIP and its various committees for applicability in patients living in middleincome and low-income countries.

\section{RESULTS}

\section{Search and study selection results}

A total of 69 articles were identified in the described databases. After eliminating the duplicates and reviews, 13 articles met the inclusion criteria and were eligible. These articles were included in the qualitative synthesis and the most relevant ones that do not include patients reported in other case series are described by their characteristics in table 1.

Although the main pathogenesis of COVID-19 may be similar to other viruses such as influenza, it has shown some clinical presentations which are different from those usually found in those classical respiratory viruses. On 24 April 2020, a new presentation of SARS-CoV-2 in children was described by Riphagen $e t$ al in the UK. ${ }^{6}$ The first report described a cohort of eight children with COVID-19 who required hospitalisation in intensive care and had an unusual clinical behaviour characterised by 


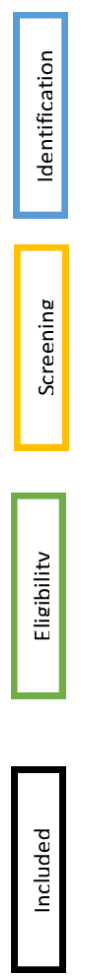

Records identified through

database searching $(n=52)$
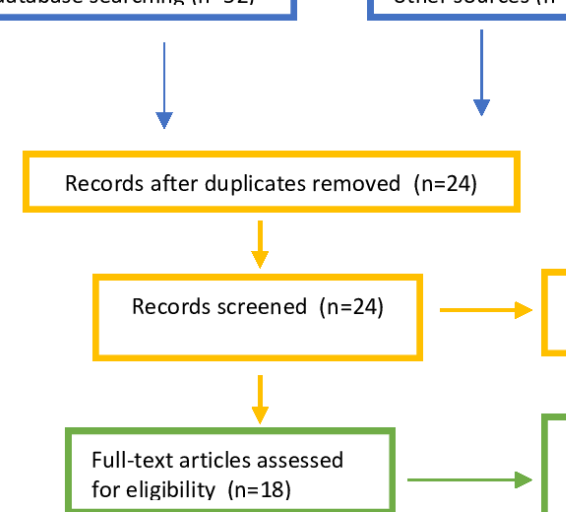

Records excluded $(n=6)$

Full-text articles articles excluded with

reasons $(n=3)$

-Population other than PIMS-TS $(n=1)$

-Full text not available $(n=1)$.

Figure 1 Selection process. We followed the PRISMA guidelines for reporting in systematic reviews and meta-analyses. PIMS-TS, paediatric inflammatory multisystem syndrome temporally associated with COVID-19; PRISMA, Preferred Reporting Items for Systematic Reviews and Meta-Analyses.

a severe hyperinflammatory state, with clinical similarity between all eight patients. ${ }^{7-9}$

The Royal College of Paediatrics and Child Health called this new entity PIMS-TS. ${ }^{10}$ Subsequently, the CDC and WHO called it multisystem inflammatory syndrome in children (MIS-C). ${ }^{11}{ }^{12}$ In general, both terms refer to the same entity, and the latter name has been the most frequently used in the main descriptions of this disease (box 1).

The largest series described to date is that of the CDC in Atlanta, with 570 patients. Using a latent class analysis statistical model, it attempted to divide the cases into three large groups according to their common clinical characteristics. ${ }^{13}$ Class I included those with symptoms that could overlap with macrophage activation syndrome, with a large inflammatory response. Class II had predominantly respiratory involvement and signs suggestive of active COVID-19 disease with a high rate of RT-PCR seropositivity (84\%). Class III had clinical manifestations that could overlap with Kawasaki disease (KD), and only $2 \%$ were RT-PCR positive. ${ }^{13}$

In this regard, most studies report that the patients have a negative RT-PCR and positive antibody or serology tests. In fact, a negative RT-PCR has been found in $40 \%$ of patients with positive antibody tests. Although RT-PCR is an imperfect test, it is considered the gold standard today. In the described series, $46 \%$ of the cases had a positive serology and a negative RT-PCR, which suggests that, in these patients, the infection occurred possibly weeks earlier. An average of $25 \%$ of the patients in the included studies had both positive serology and positive RT-PCR (online supplemental table S1).

\section{DISCUSSION}

PIMS-TS is characterised by a very significant ongoing inflammatory response, in crescendo, which in fact has been the key element in the Atlanta CDC (14 May) and WHO (15 May) definitions (box 1). ${ }^{11} 12$ Characteristically, these patients present with high leucocytosis, CRP, procalcitonin and serum ferritin. ${ }^{13}$ Hoang et $a l^{14}$ reported lower expression of circulating CD16+ CD56+ natural killer cells and more profound lymphopaenia in children with PIMS-TS compared with those without PIMS-TS.

Primarily, there is an initial innate immune response with the macrophages as the principal actors. From the pathophysiological point of view, it is striking that more than $90 \%$ of children with PIMS-TS have elevated CRP and ferritin. CRP is an acute phase reactant that usually rises after 6 hours of an inflammatory state and is produced by hepatocytes and adipose tissue in response to interleukin (IL)-1, IL-6 and TNF- $\alpha$ (tumor necrosis factor alpha) stimulation. ${ }^{15} 16$ This acute phase reactant from the pentraxin family identifies phosphatidylserine on the surface of cells that have initiated a programmed cell death pattern of apoptosis by activating the complement system. This biomarker is very useful for diagnosis and follow-up, especially in middle-income and low-income countries (given its low cost) and should be considered on admission with subsequent follow-up. 


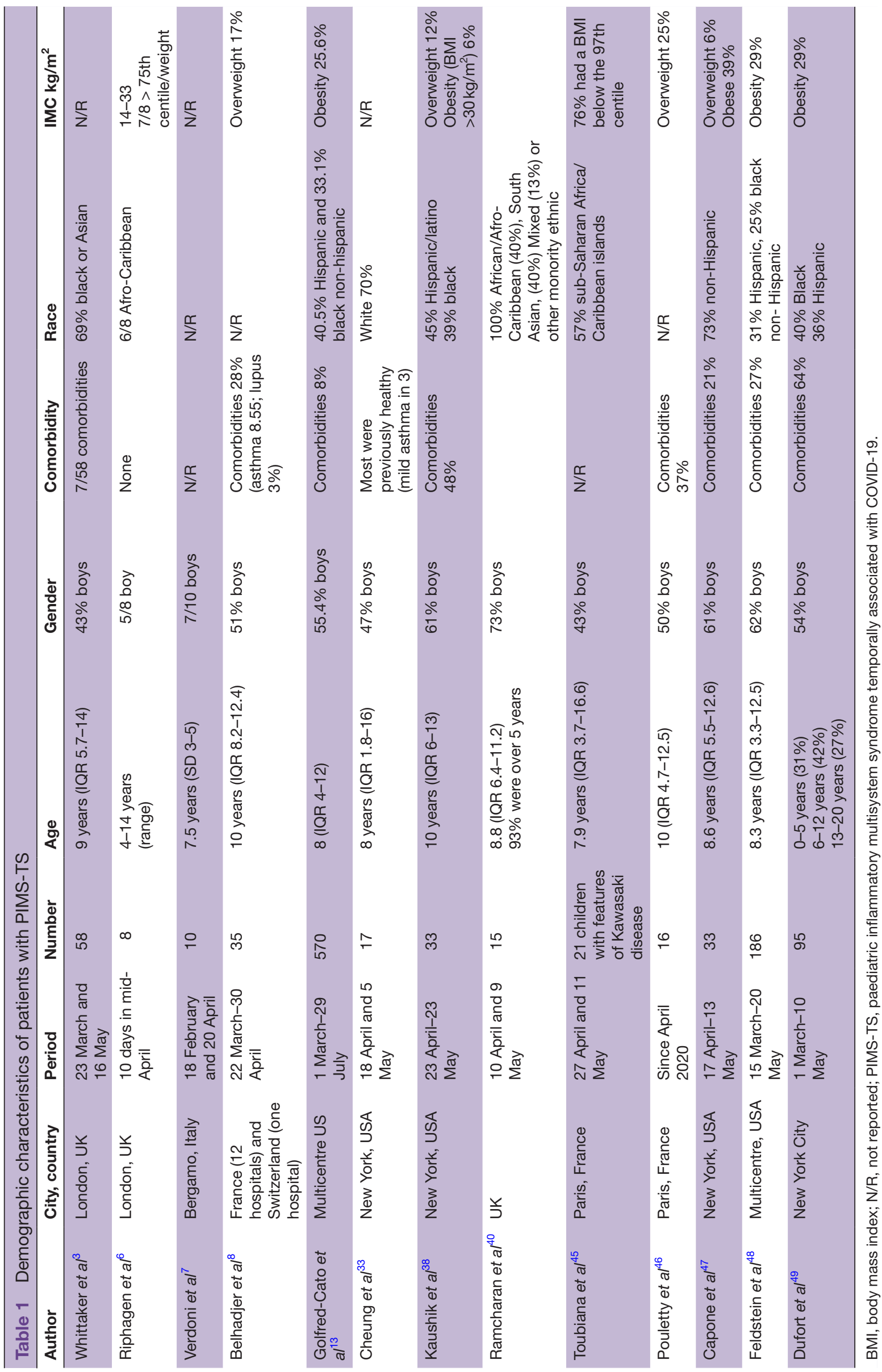

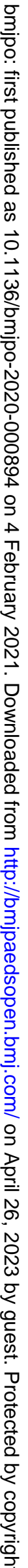


Box 1 Royal College of Paediatrics and Health Child (RCPCH), Centers for Disease Control and Prevention (CDC) and WHO definitions criteria for paediatric inflammatory multisystem syndrome temporally associated with COVID-19

\section{RCPCH definition ${ }^{10}$}

1. A child presenting with persistent fever, inflammation (neutrophilia, elevated $\mathrm{C}$ reactive protein (CRP) and lymphopaenia) and evidence of single or multiorgan dysfunction (shock, cardiac, respiratory, renal, gastrointestinal or neurological disorder) with additional features. This may include children fulfilling full or partial criteria for Kawasaki disease.

2. Exclusion of any other microbial cause, including bacterial sepsis, staphylococcal or streptococcal shock syndromes, infections associated with myocarditis such as enterovirus (waiting for results of these investigations should not delay seeking expert advice).

3. SARS-CoV-2 PCR testing may be positive or negative.

\section{CDC definition ${ }^{11}$}

1. An individual aged $<21$ years presenting with fever*, laboratory evidence of inflammationt and evidence of clinically severe illness requiring hospitalisation, with multisystem $(>2)$ organ involvement (cardiac, renal, respiratory, haematological, gastrointestinal, dermatological or neurological).

2. No alternative plausible diagnoses.

3. Positive for current or recent SARS-CoV-2 infection by RT-PCR, serology or antigen test; or COVID-19 exposure within the 4 weeks prior to the onset of symptoms.

\section{WHO definition ${ }^{12}$}

Children and adolescents $0-19$ years of age with fever $>3$ days.

And two of the following:

1. Rash or bilateral non-purulent conjunctivitis or muco-cutaneous inflammation signs (oral, hands or feet).

2. Hypotension or shock.

3. Features of myocardial dysfunction, pericarditis, valvulitis or coronary abnormalities (including ECHO findings or elevated troponin/ NT-proBNP).

4. Evidence of coagulopathy (by PT, PTT and elevated D-dimers).

5. Acute gastrointestinal problems (diarrhoea, vomiting or abdominal pain).

And

Elevated markers of inflammation such as ESR, CRP or procalcitonin.

And

No other obvious microbial cause of inflammation, including bacterial sepsis, staphylococcal or streptococcal shock syndromes.

And

Evidence of COVID-19 (RT-PCR, antigen test or serology positive) or likely contact with patients with COVID-19.

${ }^{*}$ Fever $>38.0^{\circ} \mathrm{C}$ for $\geq 24$ hours or report of subjective fever lasting $\geq 24$ hours. tIncluding, but not limited to, one or more of the following: an elevated CRP, erythrocyte sedimentation rate, fibrinogen, procalcitonin, D-dimer, ferritin, lactic acid dehydrogenase or interleukin 6 , elevated neutrophils, reduced lymphocytes and low albumin.

Additionally, a marked elevation of ferritin (2-10 times its normal value) has been observed in more than $90 \%$ of the series. ${ }^{13-17}$ Ferritin is a protein that stores iron and releases it in a controlled fashion but also in pathophysiological conditions. Its levels can reflect macrophage response to free haemoglobin as well as DNA viruses, intracellular bacterial infections and parasites. ${ }^{18-20}$ Ferritin can induce positive feedback inflammation, upregulating toll-like receptor 9 (TLR-9), which leads macrophage inflammasome IL- 1 and IL-18 to feed forward ferritin production. TLR-9 may also be stimulated by viral DNA, other infections and host damageassociated molecular patterns (DAMPs). ${ }^{20}$ This whole process generates a large number of inflammasomes and an enhanced inflammatory pathway, delivering the "cytokine storm'. This precipitate cell death with a pyroptosis pattern and new DAMPs that stimulate TLR-9. ${ }^{20}$ This was described as 'Hyperferritinemic Syndrome' by Rosário et al. ${ }^{21}$

Nevertheless, there is evidence of an unusual late adaptive immunity response. It has come to the researchers' attention that PIMS-TS occurred between 4 and 6 weeks after the peak of cases reported as positive for SARS-CoV-2 in each country had been reached. ${ }^{13}$ Pérez-Toledo $e t a l^{22}$ recently described eight patients with PIMS-TS with a negative RT-PCR but with significant elevation of $\operatorname{IgG}$ and IgA and negative IgM. Additionally, they found elevated IgG1 and IgG3 in these children, which are immunoglobulin isotypes associated with serum supplement activation. This situation is consistent with highly elevated CRP related to COVID-19, which activates the complement system. The elevation of these immunoglobulins suggests that PIMS-TS occurs due to tissue damage induced by autoantibodies, a situation that has been described in other types of coronavirus infection. ${ }^{23}{ }^{24}$ We are not aware of any studies in middle-income and low-income countries that have described this serological behaviour. Studies are needed to help clear up this aspect, especially when all the diagnostic test options for SARS-CoV-2 are not always available. In these countries with limited resources, we suggest taking an initial RT-PCR. If this is negative and there is a high index of suspicion of PIMSTS, due to the signs and symptoms, a total antibody or IgM/IgG test should be performed. ${ }^{24}$

For PIMS-TS, most of the series described coagulation disorders. Severe coagulopathy was seen in $70 \%-80 \%$ of the cases (very high D-dimers, prolonged PT and PTT). ${ }^{13}$ Like inflammation, coagulation is necessary for host defence. In addition, proinflammatory cytokines, monocytes/macrophages, neutrophil activation and extracellular neutrophil traps can foster local thrombosis. COVID-19 associated coagulopathy (CAC) is complex and in some ways pathophysiologically different from SIC. ${ }^{25} 26$

Cytokine levels of IL-1 $\beta$ and IL-6 are elevated, which induces thrombocytosis and hyperfibrinogenaemia, and the ACE-2 receptor is stimulated by SARS-CoV-2, leading to a massive release of plasminogen activators. ${ }^{27}{ }^{28}$ D-dimer levels are very high in PIMS-TS, but consumptive coagulopathy is rare in its early phase. ${ }^{28}$ Elevated D-dimer levels can be present in a wide variety of inflammatory and prothrombotic conditions ${ }^{29}$; in COVID-19, these are probably more associated with inflammation than thrombosis. ${ }^{30}$ Furthermore, serum hyaluronic acid, a key 
glycocalyx component, is higher during infancy, progressively diminishing over the years. ${ }^{19} 31$ This could explain a more protected endothelium and a lower probability of a hypercoagulable state. In addition, CAC has an overlapping pathophysiology with other coagulopathies like haemophagocytic syndrome/haemophagocytic lymphohistiocytosis, antiphospholipid syndrome and thrombotic thrombocytopaenic purpura/haemolytic uraemic syndrome, but some unique aspects make it a probably new type of coagulopathy. ${ }^{32}$

From a clinical and laboratory perspective, PIMS-TS has usually been seen in previously healthy and frequently obese $(30 \%-60 \%$ of the series) children over 8 years of age $(80 \%$ of the cases) (table 1$)$. Initially, the group from the UK found MIS-C in patients of African descent, but it has been described in patients of all origins. ${ }^{42} 33$ Persistent high fever for more than three to five consecutive days, maculopapular skin lesions (50\%-60\%) reminiscent of KD and, frequently, signs of shock at the time of presentation have been the initial clinical characteristics. ${ }^{33}$ Digestive symptoms (including nausea, vomiting, diarrhoea or abdominal pain) usually present in most cases, as well as myocardial involvement (more than $60 \%$ of the series).${ }^{143}$ Cardiac involvement is broad and variable, with features including myocardial dysfunction (100\% of the initial UK description and $60 \%$ in other series), coronary aneurysms, pericarditis, arrhythmias, refractory shock and elevated troponin I or proBNP ${ }^{32-34}$ (online supplemental table S1).

\section{Guidelines for PIMS-TS management in middle-income and low-income countries}

With regard to treatment in middle-income and lowincome countries, it is very important to maintain a high index of suspicion. Therefore, in these countries, it is important to use a systematic approach including early recognition and a bundle similar to those recommended for patients with other serious diseases. An expert consensus recently published in the UK using the Delphi method provides a good summary of the recommended treatments. ${ }^{34}$ This approach is recommended for high-income countries. Using the evidence found, we adapted these recommendations, together with those of the SCCM (Society of Critical Care Medicine) sepsis consensus ${ }^{16}$ for use in middle-income and low-income countries. We believe that a comprehensive approach to PIMS-TS patients is necessary and that taking these recommendations as a whole could have an impact on the outcomes of PIMS-TS patients in these countries.

From the first presentation to the emergency department and/or PICU, two approaches can be assumed: one general and one specific (box 2):

\section{General approach}

A comprehensive approach should be used, similar to that recommended for patients with sepsis with organ dysfunction or septic shock. In this case, the contagiousness of SARS-CoV-2 requires the use of personal
Box 2 Summary of recommendations for management of paediatric inflammatory multisystem syndrome temporally associated with COVID-19 in countries with limited

resources
A. General approach
1. Early detection.
2. Immediate, time-sensitive resuscitation
a. Oxygen therapy.
b. Fluid resuscitation.
c. Vasoactive drugs.
d. Antibiotic therapy: if bacterial coinfection is suspected.

3. Stabilisation with adequate monitoring.

4. Timely referral or transfer according to the context and available resources.

5. Continuous measurement of processes.

\section{B. Specific approach}

1. Human immunoglobulin: $2 \mathrm{~g} / \mathrm{kg}$ for moderate to severe cases.

2. Steroids:

- $1-2 \mathrm{mg} / \mathrm{kg} / \mathrm{dose}$ of methylprednisolone three or four times per day.

- High doses in cases of shock with high vasopressor requirement.

3. Anakinra:

- Only in cases refractory to steroids and IVIG (intravenous immune globulin). Not available in all countries.

4. Anticoagulation is recommended for:

a. Documented thrombosis.

b. Echocardiogram with an EF(ejection fraction) of less than $35 \%$.

5. Antiplatelet treatment: recommended for thrombocytosis $>450000 \mu / L$.

protective equipment that prevents the spread of the virus, particularly in patients with a positive RT-PCR.

Moreover, the American College of Critical Care Medicine points out the need to give more attention to institutional practice guidelines (IPGs) based on each facility's capability. ${ }^{35}$ Once IPGs are established, diagnostic and therapeutic measures known as 'patient care bundles' (PCBs) should be developed for a better approach and control of established processes. The PCBs include three to five evidence-based practices related to a healthcare process that should be performed collectively to achieve a synergistic result that improves care. ${ }^{36} 37$

1. Early detection: a comprehensive approach based on a high index of suspicion is critical. This disease may occur with a wide spectrum of symptoms, so it should be suspected in all patients with a fever lasting more than 3 days associated with the symptoms described in box 1 . Contact with a positive case is not always clear.

2. Immediate, time-sensitive resuscitation:

- Oxygen therapy: this is part of the strategies described in recent sepsis guidelines. ${ }^{16} 37$ High flow nasal cannulas and non-invasive ventilation have been considered in many reports, ${ }^{38}$ especially in patients who have a deteriorated respiratory pattern with the use of accessory muscles or an $\mathrm{SaO}_{2} / \mathrm{FiO}_{2}$ ratio less than 264. Most series describe respiratory involvement ranging from $20 \%$ to $60 \%$ (online sup- 
plemental table S1) and, generally, if endotracheal intubation is required, it is more highly associated with cardiovascular involvement. Cases classified as class II by the CDC may be classified in these groups. ${ }^{33} 38$

- Fluid resuscitation: it is important to consider the recommendations in recently published guidelines. ${ }^{16}$ In healthcare systems where staff and equipment for advanced airway management are available, up to $40-60 \mathrm{~mL} / \mathrm{kg}(10-20 \mathrm{~mL} / \mathrm{kg}$ per bolus) of balanced crystalloids (Ringer's lactate or Plasma-Lyte) can be given over the first hour, titrated to clinical markers of cardiac output and discontinued if signs of fluid overload develop. In healthcare systems without the availability of intubation, crystalloid boluses may only be given in cases of hypotension (decompensated shock); in these cases, up to $40 \mathrm{~mL} / \mathrm{kg}$ of bolus fluid $(10-20 \mathrm{~mL} / \mathrm{kg}$ per bolus) may be infused over the first hour with titration to clinical markers of cardiac output, and discontinued if signs of fluid overload develop. If the child is not hypotensive, but has compensated shock, only maintenance fluids should be started, avoiding bolus fluids that are associated with worse outcomes. ${ }^{35-38}$

- Vasoactive drugs: according to the clinical condition, most series describe the need for vasoactive drugs in $10 \%-60 \%$ of the cases with PIMS-TS. Most patients respond to fluid resuscitation. If necessary, epinephrine or norepinephrine should be considered according to the patient's condition. ${ }^{16}{ }^{39}$ Inotropes like dopamine, milrinone and levosimendan were reported to have been used in PIMS-TS. ${ }^{38-40}$

- Antibiotic therapy: if bacterial coinfection is suspected, the first dose is recommended within the first 3 hours for sepsis associated with organ dysfunction or within the first hour for children with septic shock. ${ }^{39-41}$

3. Stabilisation with adequate monitoring: if possible, advanced haemodynamic monitoring should be instituted. Cardiac ultrasound/echocardiography or $\mathrm{S}_{\text {cyo2 }}$ measurements have been suggested by recent guidelines ${ }^{16}$ and patients with PIMS-TS. ${ }^{42}$

4. Timely referral or transfer is desirable in this context. In middle-income and low-income countries, it is common for patients to be transferred to higher complexity sites. Patients who are deteriorating or who may need intensive care should be identified. In the PIMSTS of the CDC group, $84 \%$ of the cases had to be transferred to paediatric intensive care. ${ }^{1640-42}$

5. Continuous measurement of processes and corrections must be instituted for a continuous quality improvement process. ${ }^{42}$

\section{Specific approach}

It is important to emphasise that, in moderate to severe cases, the use of immunomodulatory treatment should be considered. Heterogeneous management including human immunoglobulin, systemic steroids, anakinra, tocilizumab and aspirin ${ }^{40-45}$ has been reported in the described series (online supplemental table S1). The American College of Rheumatology (ACR) recommendations for immunomodulatory therapy ${ }^{42}$ have recently been published. We sought to adapt these recommendations to middle-income and low-income countries where resources are limited and each intervention must be streamlined according to need.

- IVIG: high doses $(2 \mathrm{~g} / \mathrm{kg}$ ) should be considered for moderate to severe cases, particularly those with myocardial involvement. Prior to beginning the infusion, restored heart function must be verified. ${ }^{42}$

- Steroids: steroids have recently been shown to be useful in modifying the clinical course of the disease in adults with severe pneumonia, particularly if they are on mechanical ventilation. ${ }^{43-47}$ In patients with PIMS-TS, low doses could be considered in all cases (used in $70 \%$ of the series; online supplemental table $\mathrm{S} 1)$. Dosing schemes of $1-2 \mathrm{mg} / \mathrm{kg} /$ dose of methylprednisolone or its equivalent three or four times per day have been recommended. The ACR suggests considering high doses in cases of shock or in those with a high need for vasopressors, and we believe this recommendation is very important for middleincome and low-income countries, especially considering the frequency of late consults with advanced disease.

- Anakinra is suggested by the ACR consensus for use in cases of steroid or IVIG-refractory PIMS-TS. ${ }^{42}$ However, in many countries, its use is not approved, or it is not available, and other biological agents are used. Prospective studies are needed to evaluate the efficacy and safety of these medications in PIMS-TS.

- Anticoagulation and antiplatelet treatment: anticoagulation has become a fundamental treatment in adults, considering that there is a procoagulant and hypofibrinolytic state in severe SARS-CoV-2 infection. ${ }^{42}{ }^{47-50}$ In children with PIMS-TS, it is recommended only in cases of documented thrombosis or in patients with an echocardiogram ejection fraction less than $35 \% .{ }^{43} 47$ Aspirin would also be recommended in patients with thrombocytosis $(>450000 \mu / \mathrm{L})$ or Kawasaki-like disease criteria. ${ }^{42} 47$

The prognosis of the disease is usually good, with patient survival greater than $95 \%$ in different published series. ${ }^{564-50}$ A mortality of $1 \%-2 \%$ has been described in the published series and up to $15 \%$ with cardiovascular sequelae, including aneurysms or dysfunction. ${ }^{33} 48-50$ These patients should be followed up after discharge by interdisciplinary and multidisciplinary teams including infectious disease, rheumatology and paediatrics. However, there are incomplete data from all the cases, along with a knowledge gap regarding mild and moderate cases, the natural course and the clinical behaviour of the disease. $^{847-50}$ 
CONCLUSION

PIMS-TS is a new type of presentation of SARS-CoV-2 infection, with an exaggerated inflammatory response and inadequate inflammatory resolution with frequentbut not exclusive-digestive and myocardial involvement. It should be considered as a new disease with unique symptoms, a greater variety of clinical courses and possibly different physiological mechanisms. In middleincome and low-income countries, studies should be performed to learn more about this disease in these regions and determine if they have different phenotypic behaviours. In addition, the real role of some inflammatory biomarkers and cost-effective therapeutic strategies should be determined.

\section{Author affiliations}

${ }^{1}$ Department of Critical Care Medicine and Pediatrics Fundación Cardioinfantil Instituto de Cardiología, Universidad de la Sabana, CES Graduate School, Sepsis Committee Latin American Society of Pediatric Intensive Care (SLACIP), Bogotá, Colombia

${ }^{2}$ Pediatric Intensive Care Unit and Department of Pediatrics, Hospital Universitario da Universidad de São Paulo and Hospital Sírio Libanês, Sepsis Committee Latin American Society of Pediatric Intensive Care (SLACIP), Sao Paulo, Brazil ${ }^{3}$ Department of Pediatrics Facultad de Medicina Universidad Nacional del Nordeste, Sepsis Committee Latin American Society of Pediatric Intensive Care (SLACIP), Corrientes, Argentina

${ }^{4}$ Pediatric Intensive Care Unit. Hospital Churruca - Visca Medical Complex, Ricardo Gutiérrez Children's Hospital, Sepsis Committee. Latin American Society of Pediatric Intensive Care (SLACIP), Buenos Aires, Argentina

${ }^{5}$ Pediatric Intensive Care Unit Ricardo Gutiérrez Children's Hospital, Sepsis Committee Latin American Society of Pediatric Intensive Care (SLACIP), Buenos Aires, Argentina

${ }^{6}$ Pediatric Intensive Care Unit Ricardo Gutiérrez Children's Hospital, Sepsis Committee. Latin American Society of Pediatric Intensive Care (SLACIP), Buenos Aires, Argentina

\section{Twitter Jaime Fernández-Sarmiento @jfersar and Daniela De Souza @danicds72}

Contributors All authors conceptualised and designed the literature search. JF-S, DDS and RJ initiated the search and a first draft. All authors contributed to subsequent drafts. JF-S, as group leader, supervised and moderated the search, initial drafts, the overall collation of the figures and tables and final manuscript. All authors approved the final manuscript as submitted and agree to be accountable for all aspects of the work.

Funding The authors have not declared a specific grant for this research from any funding agency in the public, commercial or not-for-profit sectors.

Competing interests None declared.

Patient consent for publication Not required.

Provenance and peer review Not commissioned; externally peer reviewed.

Data availability statement All data relevant to the study are included in the article or uploaded as supplementary information. Not applicable.

Supplemental material This content has been supplied by the author(s). It has not been vetted by BMJ Publishing Group Limited (BMJ) and may not have been peer-reviewed. Any opinions or recommendations discussed are solely those of the author(s) and are not endorsed by BMJ. BMJ disclaims all liability and responsibility arising from any reliance placed on the content. Where the content includes any translated material, BMJ does not warrant the accuracy and reliability of the translations (including but not limited to local regulations, clinical guidelines, terminology, drug names and drug dosages), and is not responsible for any error and/or omissions arising from translation and adaptation or otherwise.

Open access This is an open access article distributed in accordance with the Creative Commons Attribution Non Commercial (CC BY-NC 4.0) license, which permits others to distribute, remix, adapt, build upon this work non-commercially, and license their derivative works on different terms, provided the original work is properly cited, appropriate credit is given, any changes made indicated, and the use is non-commercial. See: http://creativecommons.org/licenses/by-nc/4.0/.

ORCID iD

Jaime Fernández-Sarmiento http://orcid.org/0000-0003-2874-2949

\section{REFERENCES}

1 World Health Organization. Coronavirus disease 2019 (COVID-19): situation report. Available: http://www.who.int/docs/default-source/ coronaviruse/situation-reports.2 [Accessed 28 Jun 2020].

2 Yang X, Yu Y, Xu J, et al. Clinical course and outcomes of critically ill patients with SARS-CoV-2 pneumonia in Wuhan, China: a singlecentered, retrospective, observational study. Lancet Respir Med 2020;8:475-81.

3 Whittaker E, Bamford A, Kenny J, et al. Clinical characteristics of 58 children with a pediatric inflammatory multisystem syndrome temporally associated with SARS-CoV-2. JAMA 2020;324:259-69.

4 González-Dambrauskas S, Vásquez-Hoyos P, Camporesi A, et al. Pediatric critical care and COVID-19. Pediatrics 2020;146:e20201766.

5 DeBiasi RL, Song X, Delaney M, et al. Severe coronavirus Disease-2019 in children and young adults in the Washington, DC, metropolitan region. J Pediatr 2020;223:199-203.

6 Riphagen S, Gomez X, Gonzalez-Martinez C, et al. Hyperinflammatory shock in children during COVID-19 pandemic. Lancet 2020;395:1607-8.

7 Verdoni L, Mazza A, Gervasoni A, et al. An outbreak of severe Kawasaki-like disease at the Italian epicentre of the SARS-CoV-2 epidemic: an observational cohort study. Lancet 2020;395:1771-8.

8 Belhadjer Z, Méot M, Bajolle F, et al. Acute heart failure in multisystem inflammatory syndrome in children in the context of global SARS-CoV-2 pandemic. Circulation 2020;142:429-36.

9 Grimaud M, Starck J, Levy M, et al. Acute myocarditis and multisystem inflammatory emerging disease following SARS-CoV-2 infection in critically ill children. Ann Intensive Care 2020;10:69.

$10 \mathrm{RCPCH}$. Royal College of paediatrics and child health. Available: https://www.rcpch.ac.uk/resources/guidance-paediatricmultisystem-inflammatory-syndrome-temporally-associated-covid19-pims

11 CDC. Multisystem inflammatory syndrome in children (MIS-C) associated with coronavirus disease 2019 (COVID-19). Available: https://emergency.cdc.gov/han/2020/han00432.asp

12 WHO. Multisystem inflammatory syndrome in children and adolescents with COVID 19 scientific brief. Available: https://www. who.int/publications-detail/multisystem-inflammatory-syndrome-inchildren-and-adolescents-with-covid-19 [Accessed 15 May 2020].

13 Golfred-Cato S, Bryant B, Leung J. COVID-19- associated Multiystem inflammatory syndrome in Children- United States, March-July 2020, 2020. Available: https://www.cdc.gov/mmwr/ volumes/69/wr/pdfs/mm6932e2-H.pdf

14 Hoang A, Chorath K, Moreira A, et al. COVID-19 in 7780 pediatric patients: a systematic review. EClinicalMedicine 2020;24:100433.

15 Tan L, Wang Q, Zhang D, et al. Lymphopenia predicts disease severity of COVID-19: a descriptive and predictive study. Sig Transduct Target Ther 2020;5.

16 Weiss SL, Peters MJ, Alhazzani W, et al. Surviving sepsis campaign international guidelines for the management of septic shock and sepsis-associated organ dysfunction in children. Intensive Care Med 2020;46:10-67.

17 Alhazzani W, Møller MH, Arabi YM, et al. Surviving sepsis campaign: guidelines on the management of critically ill adults with coronavirus disease 2019 (COVID-19). Intensive Care Med 2020;46:854-87.

18 Jaume M, Yip MS, Cheung CY, et al. Anti-Severe acute respiratory syndrome coronavirus spike antibodies trigger infection of human immune cells via a pH- and cysteine protease-independent FcyR pathway. J Virol 2011;85:10582-97.

19 Fernández-Sarmiento J, Salazar-Peláez LM, Carcillo JA. The endothelial glycocalyx: a fundamental determinant of vascular permeability in sepsis. Pediatr Crit Care Med 2020;21:e291-e300.

20 Carcillo JA, Kernan KK, Horvat CM, et al. Why and how is Hyperferritinemic sepsis different from sepsis without Hyperferritinemia? Pediatr Crit Care Med 2020;21:509-12.

21 Rosário C, Zandman-Goddard G, Meyron-Holtz EG, et al. The hyperferritinemic syndrome: macrophage activation syndrome, still's disease, septic shock and catastrophic antiphospholipid syndrome. BMC Med 2013;11:185

22 Pérez-Toledo M, Faustini S, Jossi S. Serology confirms SARSCoV-2 infection in PCR-negative children presenting with pediatric inflammatory multi-system syndrome. medRxiv 2020. 
23 Kam YW, Kien F, Roberts A, et al. Antibodies against trimeric S glycoprotein protect hamsters against SARS-CoV challenge despite their capacity to mediate FcgammaRII-dependent entry into B cells in vitro. Vaccine 2007:25:729-40.

24 Wang S-F, Tseng S-P, Yen C-H, et al. Antibody-dependent SARS coronavirus infection is mediated by antibodies against spike proteins. Biochem Biophys Res Commun 2014;451:208-14.

$25 \mathrm{Iba}$ T, Levy JH, Connors JM, et al. The unique characteristics of COVID-19 coagulopathy. Crit Care 2020;24:360.

26 Iba T, Levy JH. Sepsis-induced coagulopathy and disseminated intravascular coagulation. Anesthesiology 2020;132:1238-45.

27 Waltuch T, Gill P, Zinns LE, et al. Features of COVID-19 postinfectious cytokine release syndrome in children presenting to the emergency department. Am J Emerg Med 2020;38:2246.e3-2246. e6.

28 Varga Z, Flammer AJ, Steiger $\mathrm{P}$, et al. Endothelial cell infection and endotheliitis in COVID-19. Lancet 2020;395:1417-8.

29 Yu B, Li X, Chen J, et al. Evaluation of variation in D-dimer levels among COVID-19 and bacterial pneumonia: a retrospective analysis. J Thromb Thrombolysis 2020;50:1-10.

30 Bates SM. D-Dimer assays in diagnosis and management of thrombotic and bleeding disorders. Semin Thromb Hemost 2012;38:673-82

31 Trivedi P, Cheeseman P, Mowat AP. Serum hyaluronic acid in healthy infants and children and its value as a marker of progressive hepatobiliary disease starting in infancy. Clin Chim Acta 1993;215:29-39.

32 Tan W, Aboulhosn J. The cardiovascular burden of coronavirus disease 2019 (COVID-19) with a focus on congenital heart disease. Int J Cardiol 2020;309:70-7.

33 Cheung EW, Zachariah P, Gorelik M, et al. Multisystem inflammatory syndrome related to COVID-19 in previously healthy children and adolescents in New York City. JAMA 2020;324:294.

34 Harwood R, Allin B, Jones CE, et al. A national consensus management pathway for paediatric inflammatory multisystem syndrome temporally associated with COVID-19 (PIMS-TS): results of a national Delphi process. Lancet Child Adolesc Health 202010.1016/S2352-4642(20)30304-7. [Epub ahead of print: 18 Sep 2020].

35 Davis AL, Carcillo JA, Aneja RK, et al. American College of critical care medicine clinical practice parameters for hemodynamic support of pediatric and neonatal septic shock. Crit Care Med 2017;45:1061-93.

36 Wip C, Napolitano L. Bundles to prevent ventilator-associated pneumonia: how valuable are they? Curr Opin Infect Dis 2009;22:159-66.

37 Kohn Loncarica G, Fustiñana A, Jabornisky R. Recommendations for the management of pediatric septic shock in the first hour (Part one). Arch Argent Pediatr 2019;117:e14-23.

38 Kaushik S, Aydin SI, Derespina KR. Multisystem inflammatory syndrome in children (MIS-C) associated with SARS-CoV-2 infection: a multi-institutional study from New York City. J Pediatr 2020;224:24-9.
39 National Institute for Health and Care Excellence. Sepsis: recognition, diagnosis and early management. London, 2016. Available: https://www.nice.org.uk/guidance/ng51/resources/sepsisrecognition-diagnosis-and-early-manage- ment-pdf-1837508256709 [Accessed 27 Jun 2020].

40 Ramcharan T, Nolan O, Lai CY, et al. Paediatric inflammatory multisystem syndrome: temporally associated with SARSCoV-2 (PIMS-TS): cardiac features, management and short-term outcomes at a UK tertiary paediatric Hospital. Pediatr Cardiol 2020;41:1391-401.

41 Fernández-Sarmiento J, Carcillo JA, Salinas CM, et al. Effect of a sepsis educational intervention on hospital stay. Pediatr Crit Care Med 2018;19:e321-8.

42 American College of Rheumatology. Clinical guidance for pediatric patients with multisystem inflammatory syndrome in children (MIS-C) associated with SARS-CoV-2 and hyperinflammation in covid-19. Available: https://www.rheumatology.org/announcements\#Pediatri cClinicalGuidance [Accessed 17 Jun 2020].

43 RECOVERY (Randomised Evaluation of COVID-19 Therapy) University of Oxford. Low-cost dexamethasone reduces death by up to one third in hospitalized patients with severe respiratory complications of COVID-19 [Internet], 2020. Available: https://www. recoverytrial.net/news/low-cost-dexamethasone-reduces-death-byup-to-one-third-in-hospitalised-patients-with-severe-respiratorycomplications-of-covid-19 [Accessed 18 Jun 2020].

44 Rajasekaran S, Kruse K, Kovey K, et al. Therapeutic role of anakinra, an interleukin-1 receptor antagonist, in the management of secondary hemophagocytic lymphohistiocytosis/sepsis/multiple organ dysfunction/macrophage activating syndrome in critically ill children*. Pediatr Crit Care Med 2014;15:401-8.

45 Toubiana J, Poirault C, Corsia A, et al. Kawasaki-like multisystem inflammatory syndrome in children during the covid-19 pandemic in Paris, France: prospective observational study. BMJ 2020;369:m2094.

46 Pouletty M, Borocco C, Ouldali N, et al. Paediatric multisystem inflammatory syndrome temporally associated with SARS-CoV-2 mimicking Kawasaki disease (Kawa-COVID-19): a multicentre cohort. Ann Rheum Dis 2020;79:1-8.

47 Capone CA, Subramony A, Sweberg T. Characteristics, cardiac involvement and outcomes of multisystem inflammatory disease of childhood (MIS-C) associated with SARS-CoV-2 infection. J Pediatr 2020;224:141-5.

48 Feldstein LR, Rose EB, Horwitz SM, Horwitz JP, et al. Multisystem inflammatory syndrome in U.S. children and adolescents. $N$ Engl J Med 2020;383:334-46.

49 Dufort EM, Koumans EH, Chow EJ, et al. Multisystem inflammatory syndrome in children in New York state. N Engl J Med 2020;383:347-58.

50 Prata-Barbosa A, Lima-Setta F, Santos GRD, et al. Pediatric patients with COVID-19 admitted to intensive care units in Brazil: a prospective multicenter study. J Pediatr 2020;96:582-92. 